\title{
Entwicklung bäuerlicher Betriebe und landwirtschaftliche Ausbildungsverhältnisse an Sprach- und politischen Grenzen im Schweizer Jura
}

Die Daten wurden an den Landwirtschaftsschulen Ebenrain (BL), Laufen (BE) und Courtemelon (JU) gewonnen, statistisch verarbeitet und in Beziehung zu amtlich erhobenen und in den eidgenössischen Quellenwerken publizierten Angaben gesetzt. Die eigenen Erhebungen und Auswertungen ließen eine Begleitstudie zur wissenschaftlichen Arbeit «Agrargeographische Analyse im Raum der deutsch-französischen Sprachgrenze im nördlichen schweizerischen Juras entstehen (Publikation als Dissertationsarbeit im Winter 83/84). Die Studie dient der größerräumlichen Bestätigung bzw. Verwerfung von in sechs Testgemeinden des Laufentals und des Bassin de Delémont beobachteten sprachgebietlich unterschiedlichen Eigenheiten im Bereich der Landwirtschaft. Der Bezug zum eigenen Feldarbeitsgebiet wird erst in der Dissertationspublikation veröffentlicht.

Es interessierte, ob sich der Anteil der Schulabsolventen je nach Zugehörigkeit zu einer bestimmten Sprachgruppe verändert. Nur diejenigen Schulabsolventen wurden berücksichtigt, die in einer der drei obgenannten Landwirtschaftsschulen ausgebildet wurden und deren zukünftige Arbeitsstätten sich in den nachfolgend aufgeführten Bezirken befinden. In denselben Bezirken mußte der Elternwohnort sein.

\section{Die Schulungsbedingungen für Landwirte in den Kantonen Baselland, Bern und Jura}

Erfaßt wurden die Bezirke Courtelary, Moutier (beide BE), Delémont, Franches Montagnes, Porrentruy (alle drei JU), Laufen (BE), Arlesheim, Liestal Sissach, Waldenburg (alle vier BL) und Dorneck und Thierstein (beide SO).

In die Untersuchung wurde die Schule Courtemelon (kantonal, JU), Laufen (Bezirk, BE) und SissachEbenrain (kantonal, BL) einbezogen. In früheren Jahren waren nur ein oder zwei Winterkurse üblich, heute sind die Schulen eigentliche Ausbildungsstätten.

Courtemelon (Delémont): Gleichzeitig und nebeneinander wird die freiwillige Berufsschule mit Abschlußprüfung für Lehrlinge und die obligatorische Berufsschule ohne Abschlußprüfung geführt, die alle angehenden Bauern besuchen müssen. Dauer: 2 Jahre.
Ferner existiert eine ebenfalls zwei Jahre dauernde Fachschule, deren Besuch freiwillig ist. Daten für 1975/4-1981/2.

Laufen: Die Landwirtschaftsschule (Fortbildungsschule, aber auch Ausbildung von 16- bis 19jährigen) wechselte im Gebiet des Laufener Beckens den Standort mehrmals; sie befand sich im solothurnischen Büsserach, dann im bernischen Duggingen, schließlich in Laufen. Sie entspricht am ehesten der jurassischen Berufsschule ohne eigentliche Lehrabschlußprüfung. Daten für 1971-1980/81.

Ebenrain (Sissach): Obligatorisch ist heute vor dem Besuch der Fachschule die Berufsschule, die aus einer zweijährigen Lehre beim Mann und einer einjährigen bei der Frau besteht. Daten für die Jahre 1974-82.

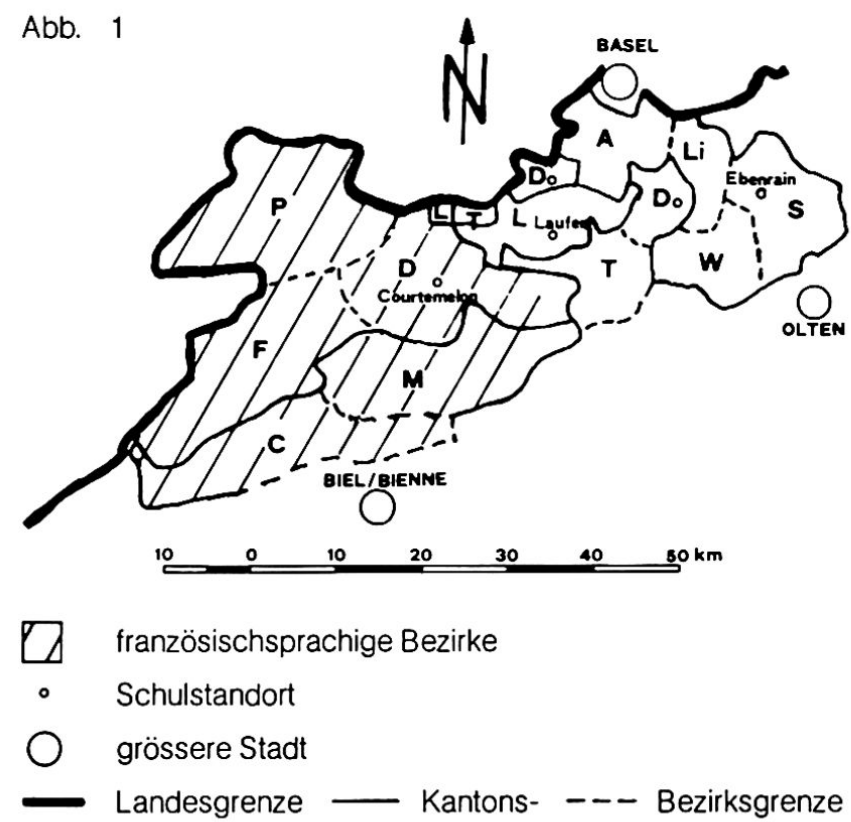

Bezirke:

D Delémont, F Franches Montagnes, P Porrentruy, C Courtelary, M Moutier, L Laufen, A Arlesheim, Li Liestal, S Sissach, W Waldenburg, Do Dorneck, T Thierstein

Kurt Wasmer-Ramer, Merkurstr. 97, 4123 Allschwil 


\subsection{Entwicklung der Anzahl der Landwirtschaftsbe- triebe in der Nordwestschweiz zwischen 1969 und 1980}

Im Folgenden wird kurz die Abb. 2 erläutert, um den Vergleich zwischen den einzelnen Bezirken unterschiedlicher Sprache und politischer Zugehörigkeit verständlich zu gestalten.

Bezirke des Kantons Jura: Delémont und die Franches Mtgnes hatten 1980 mehr Betriebe als 1975, man beachte hier die Kantonsgründung!

Die Gesamtzahl der Betriebe sank aber von 771 auf 691 , d. h. um 10,4\% in Delémont und von 556 auf 532, d. h. um 4,3\% in den Franches Mtgnes und um 22,1\% in Porrentruy. Obwohl Porrentruy keinen Landgewinn durch die Kantonsänderung erfuhr, ist die Abnahme also verhältnismäßig gering. Beim Anteil hauptberuflicher Landwirte zeigen sich ebenso relativ geringe Veränderungen: $-4,5 \%$ (Delémont), $+2,1 \%$ (Franches Montagnes), - 19,1\% (Porrentruy). Daraus folgt eine schwächere Stellung der Nebenerwerbsbetriebe. Ihr Anteil ist gesamthaft allerdings eher klein mit durchschnittlich $33,4 \%$ in Delémont, $17,5 \%$ in den Franches Mtgnes und 34,8\% im Pruntruter Zipfel.

Courtelary, Moutier (BE): Die Gesamtzahl der Landwirtschaftsbetriebe zeigt eine Abnahme von 670 auf 577 Betriebe in Courtelary und von 828 auf 510
Betriebe in Moutier. In Courtelary entspricht dies einer Verringerung um 13,9\%, in Moutier um 39,8\%, allerdings sind hier die Verluste z. T. durch kommunale Kantonswechsel bewirkt (Bsp. Mervelier, Châtillon). Rund 31,6\% beträgt die Abnahme der Haupterwerbsbetriebe in Courtelary, $43,7 \%$ in Moutier. Der Anteil der Nebenerwerbsbauern ist hier kleiner als in den deutschsprachigen Bezirken, er beträgt in Courtelary im Mittel der Jahre 1969-80 nur 21,5\%, in Moutier $32,8 \%$.

Bernischer Bezirk Laufen: Es zeigt sich von 1969 bis 1980 eine Abnahme der Gesamtheit der Betriebe von 312 auf 238 (1980 inkl. Roggenburg) oder um 23,7\%; die Haupterwerbsbetriebe gingen um 12,5\% auf 119 Betriebe zurück. 54\%(!) aller Betriebe sind Nebenerwerbsbetriebe (Ne-Betriebe), die offenbar rascher aufgeben.

Basellandschaftliche Bezirke: Zur Abnahme aller Betriebe: in Arlesheim beträgt sie 0,9\%, in Liestal $23,5 \%$, in Sissach $12,2 \%$ und in Waldenburg $16,5 \%$.

Die Haupterwerbsbetriebe vermindern sich im selben Zeitraum (1969-80) in Arlesheim um 22,9\%, in Liestal um $24,5 \%$, in Sissach um 23,6\% und in Waldenburg um $18,5 \%$. Rund $46,2 \%$ bzw. $51,9 \%$ bzw. $37,7 \%$ bzw. $38,7 \%$ der Betriebe sind Ne-Betriebe.

Abb. 2 Anzahl landwirtschaftliche Betriebe

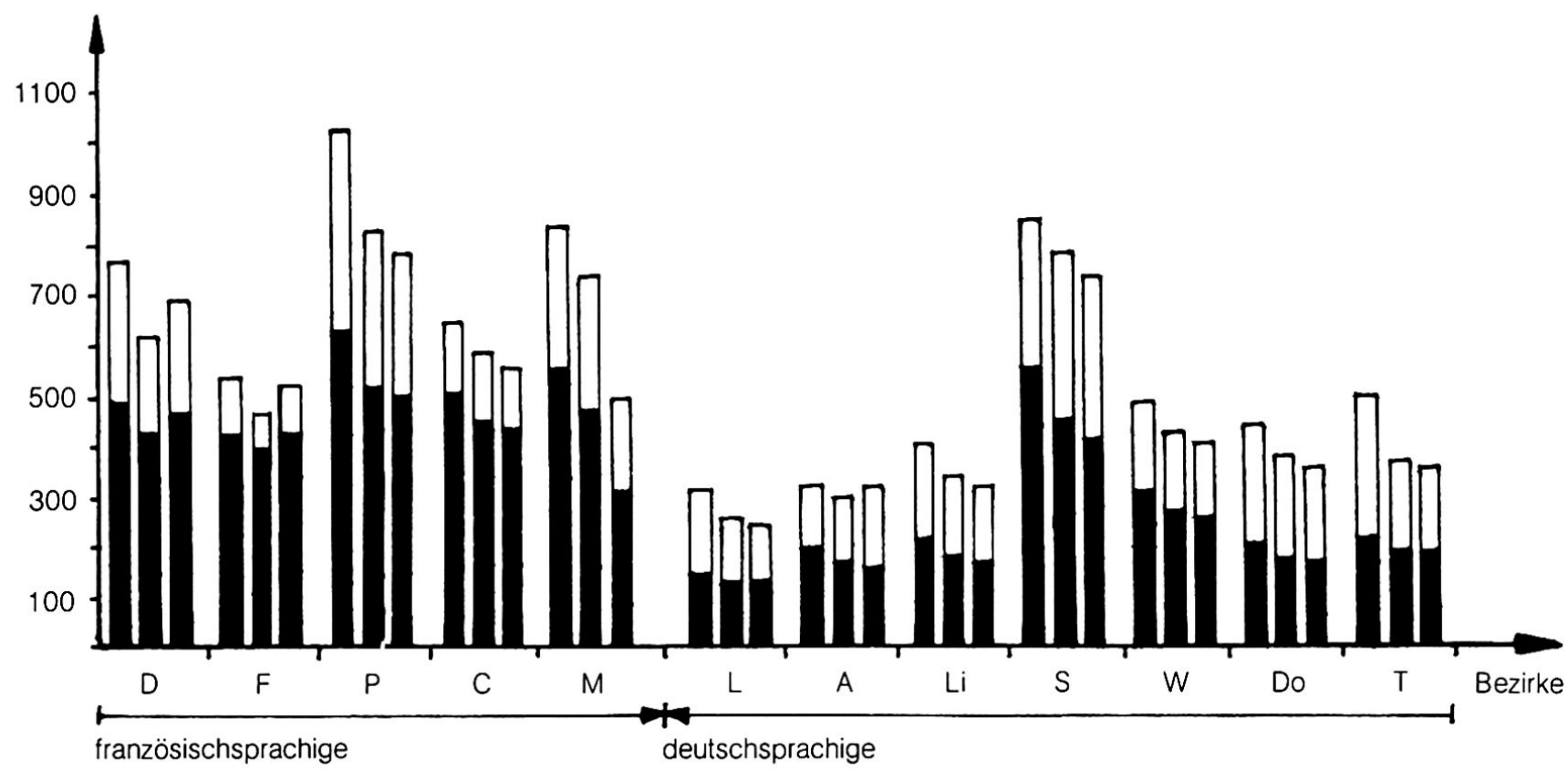

Total der Betriebe

davon im Hauptwerwerb

1. Säule Eidg. Landwirtschaftszählung 1969 (2. Teil)

2. Säule Eidg. Landwirtschaftszählung 1975 (1. Teil)

3. Säule Zählung der Gemeinden 1980

Abkürzungen der Bezirke auf Abb. 1 
Solothurnische Bezirke: Dorneck zeigte eine Abnahme von 455 auf 363 oder um 20,2\%, Thierstein um $29,2 \%$ auf 358. Die Haupterwerbsbetriebe zeigten eine Verminderung um $23,1 \%$ in Dorneck bzw. $12,4 \%$ in Thierstein.

Im Bezirk Dorneck sind 57,9\% aller Betriebe NeBetriebe, im Bezirk Thierstein 55, $1 \%$.

\subsection{Vergleich französischsprachiger bernischer mit jurassischen Bezirken und deutschsprachiger Bezirke der Kantone Baselland, Solothurn und Bern}

Im ersten Vergleich ist der Anteil von Ne-Betrieben interessant: Rund 30\% der Betriebe des Kantons Jura im Mittel der Jahre 1969, 1975 und 1980 sind NeBetriebe, rund $27,5 \%$ in den beiden französischsprachigen Bezirken des Kantons Bern; der Anteil ist also erstens annähernd gleich und zweitens betragen die Anteile weniger als einen Drittel.

Die entsprechenden Werte für den bernischen Bezirk Laufen und die solothurnischen Bezirke lauten 55,8\% und für die vier basellandschaftlichen Bezirke $42,1 \%$. Es ergeben sich für die welschen und deutschsprachigen Bezirke unabhängig von der Anzahl der Betriebe, der Bezirksfläche und damit der Dichte sprachspezifische Verhältnisse.

In der Gegenüberstellung der fünf französischsprachigen und der sieben deutschsprachigen Bezirke zeigen erstere zwischen 1969 und 1980 gesamthaft eine Abnahme von $19,3 \%$, die zweiten eine solche von $17,1 \%$. Damit ergibt sich also kaum ein Unterschied, im Gegensatz zur Entwicklung der Ne-Betriebe. Diese gingen in den französischsprachigen Bezirken von $30,3 \%$ (Anteil 1969) auf 28,4\% leicht zurück und weisen einen geringen Anteil auf, während sie in den deutschsprachigen Vergleichsbezirken zwischen 1969 und 1980 von $46,1 \%$ auf $47,8 \%$ noch eine Steigerung erfuhren und allgemein höhere Anteile zeigen.

Zusammenfassung und Folgerung: Die Anzahl Landwirtschaftsbetriebe wurde für die Stichjahre 1969, 1975 und 1980 herangezogen, um die Entwicklung von Haupt- und Nebenerwerbsbetrieben in den einzelnen Bezirken aufzuzeigen und sprachgebietliche Besonderheiten herauszuarbeiten.

In den drei Bezirken des Kantons Jura war die Abnahme der Betriebe gering, ebenso im bernischen französischsprachigen Bezirk Courtelary, nicht aber in Moutier. In den deutschsprachigen Bezirken war die Abnahme im Kanton Baselland gering, in den solothurnischen Bezirken und im Laufental dagegen mit rund einem Viertel hoch. Der Rückgang der Haupterwerbsbetriebe fiel für den Kanton Jura gering aus, was allerdings durch den Wechsel verschiedener Gemeinden bei Kantonsgründung mitbedingt ist. In den bernisch-französischsprachigen Gebieten war der Rückgang hoch, dagegen im Bezirk Laufen mit 12,5\% tief. Die Nebenerwerbsbetriebe waren durchwegs in
Abb. 3 Abnahme der Betriebe und Anteil der Nebenerwerbsbetriebe

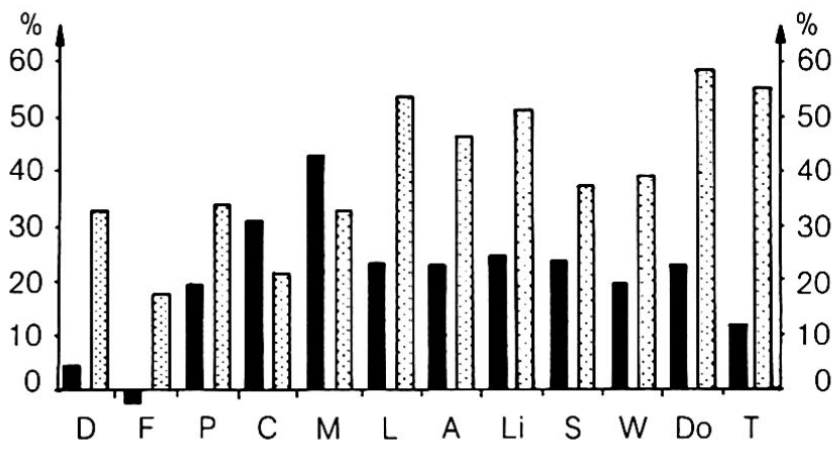

Relative Abnahme der Haupterwerbsbetriebe (Mittel der Zählungen 1969/1975/80)

圈 Relativer Anteil der Nebenerwerbsbetriebe (Mittel der Zählungen 1969/1975/80)

Abkürzungen der Bezirke auf Abb. 1.

den welschen Bezirken im Vergleich zu den deutschsprachigen untervertreten; in der Deutschschweiz erfuhren sie z.T. von 1969 bis 1980 gar eine prozentuale Steigerung.

\section{Schüleranteile in Courtemelon, Ebenrain und Laufen}

Der Schulungsgrad der Landwirte aus den genannten Gebieten kann nur annähernd bestimmt werden, da die Landwirte oft gesetzlich nicht an den Schulbesuch sowie auch einen bestimmten Schulungsort gebunden sind. Zudem ist - wie im Kanton Jura - zwar heute für Jungbauern der Berufsschulbesuch obligatorisch erklärt, jedoch ist eine Abschlußprüfung nicht Bedingung. Die Absenzenliste ist relativ hoch.

Im Folgenden wird die durchschnittliche Anzahl der Landwirte der Jahre 1969, 1975 und 1980 mit der mittleren Zahl der Schüler verglichen, und zwar für die Lehrzeit (Bauer und Bäuerin) oder die Berufs- und Fachschule an den Ausbildungsstätten Courtemelon (Delémont), Laufen und Ebenrain (Sissach). Gespräche mit Lehrern dieser Schulen klärten Fragen zur Begriffsvielfalt der Ausbildungsstufen und «Schulfreudigkeit) aus bestimmten Gegenden und Bevölkerungsschichten.

Das Laufental wäre seit 1971 an die Schule Courtemelon angeschlossen, wo Lehren absolviert werden können; wohl aus sprachlichen Gründen wurde jedoch eine eigene, allerdings sehr bescheiden ausgestattete Schule in Laufen gegründet. Zirka drei bis fünf angehende Landwirte (zwischen 16 und 19 Jahre alt) nehmen jährlich teil, sie brauchen dann auch die Fortbildungskurse nicht mehr zu besuchen. 
In Ebenrain, der basellandschaftlichen kantonalen Landwirtschaftsschule, fiel besonders der hohe Anteil von Auszubildenden aus nichtbäuerlichen und vor allem städtischen Kreisen auf. Ferner überraschte die hohe Schülerzahl für diesen Industriekanton.

In Courtemelon sehen einige Lehrer das größte Problem landwirtschaftlicher Schulen darin, daß die Motivation zum Schulbesuch bei älteren Landwirten und vor allem bei Söhnen traditioneller Bauern oft fehlt, da der Sinn der Schulung nicht erkannt würde. So wurde zwar erreicht, daß der Besuch der Berufsschule gesetzlich als obligatorisch erklärt wurde, doch beenden rund zwei Drittel der jungen Absolventen die Ausbildung ohne Fremdlehrjahr und Schlußprüfung. Die Söhne würden zu Hause stark in den Betrieb eingespannt; sie arbeiten in erster Linie für Betriebsaufstockungen auf dem Hof und werden dadurch vom Schulbesuch abgehalten. Nach einem Informanten besteht zudem im Kanton Jura und im Bezirk Laufen offenbar kein Bedürfnis nach einer Bäuerinnenschule, denn für die Jahr 1973 bis 1981/2 waren lediglich 2 Berufsschülerinnen zu registrieren! Die Ausbildung männlicher Lehrlinge aus dem Laufental in Courtemelon liegt bei rund 0,2\% der Gesamtheit der Betriebsleiter (die Prozentangaben geben den Jahresdurchschnitt des Zeitraumes 1971 bzw. 1973/4 bis 1981/82 wieder)'. Dagegen bildeten sich rund $1,35 \%$ der Betriebsleiter an der Laufener Schule weiter aus (z.T. Lehre), weitere $0,1 \%$ besuchten die Schule Ebenrain. Mädchen begannen im untersuchten Zeitraum keine Lehre. In den benachbarten Solothurner Bezirken Dorneck und Thierstein liegen die prozentualen Anteile der Lehrlinge gleich (bei beträchtlich mehr Betrieben), auch hier werden Mädchen nicht zur Ausbildung geschickt. Knapp 0,2\% bzw. $0,1 \%$ der Jungbauern besuchten die Sissacher Fachschule, kein angehender Landwirt suchte Delémont auf. Die drei kantonaljurassischen Bezirke weisen dagegen bei einer markant höheren Anzahl von Landwirtschaftsbetrieben auch hohe prozentuale Anteile von Ausgebildeten auf: obwohl kein Mädchen eine Lehre absolvierte, liegt der Anteil der jüngsten Schulabsolventen (Lehrlinge v. a. mit Lehrabschluß) bei $0,54 \%$ für Delémont, bei $0,64 \%$ für die Franches Mtgnes und bei $0,3 \%$ für Porrentruy. Die Berufsschule Courtemelon besuchten 1,43\% bzw. $1,82 \%$ bzw. $1,88 \%$ der jeweiligen Gesamtheit von Betriebsleitern. Der basellandschaftlichen Fachschule entsprechende Kurse belegten weitere $1,45 \%$ bzw. $0,74 \%$ bzw. $0,93 \%$. Damit weist der Bezirk Delémont jährlich 3,44\% neu aus- oder weitergebildete Landwirte auf, für die Franches Mtgnes sind es 3,2\%, für Porrentruy 3,11\%.

\footnotetext{
'Die durchschnittliche Schülerzahl in diesem Zeitraum wurde im Verhältnis zum Mittel der Landwirtschaftsbetriebe der Jahre 1969, 1975 und 1980 betrachtet.
}

Obwohl etliche Bauern sowohl Lehre wie Fachschule besuchen, darf doch erwartet werden, daß innerhalb von 35 bis 40 Jahren, also innerhalb einer Betriebsleitergeneration, als Folge des heutigen Schulungssystems im Kt. Jura jeder eine Grundausbildung erhält.

Die bernisch-jurassischen Bezirke Courtelary und Moutier zeigen durchwegs sehr niedrige Schülerzahlen. Mit gesamthaft 0,4\% Ausgebildeten aus Courtelary und $0,65 \%$ aus Moutier liegen sie zwar deutlich vor den solothurnischen, aber noch hinter den Laufentaler Bauern (1,64\%). Besonders markant ist der Schülerrückgang seit der Gründung des Kantons Jura 1978. Kein Bauer besuchte mehr die Berufsschule, die Lehrlingsanzahl sank von 15 auf 1 . Dagegen besuchen zahlreiche Schüler - allerdings auch solche aus dem Kanton Jura - die Schulen in Rüti (BE) und Cernier (NE).

Den höchsten Anteil ausgebildeter Landwirte verzeichnet der Kt. Baselland, wo aus dem Bezirk Arlesheim die größte Anzahl junger Männer, die $4,64 \%$ der Betriebsleiter entspricht, eine Lehre machte (manche der aus nichtbäuerlichen Kreisen stammenden Lehrlinge können später kaum einen Betrieb übernehmen, Mitt. Sekr. Landwirtschaftsschule Eben-

Abb. 4 Anzahl der Ausgebildeten pro Jahr im Vergleich des aktiven Berufsstandes

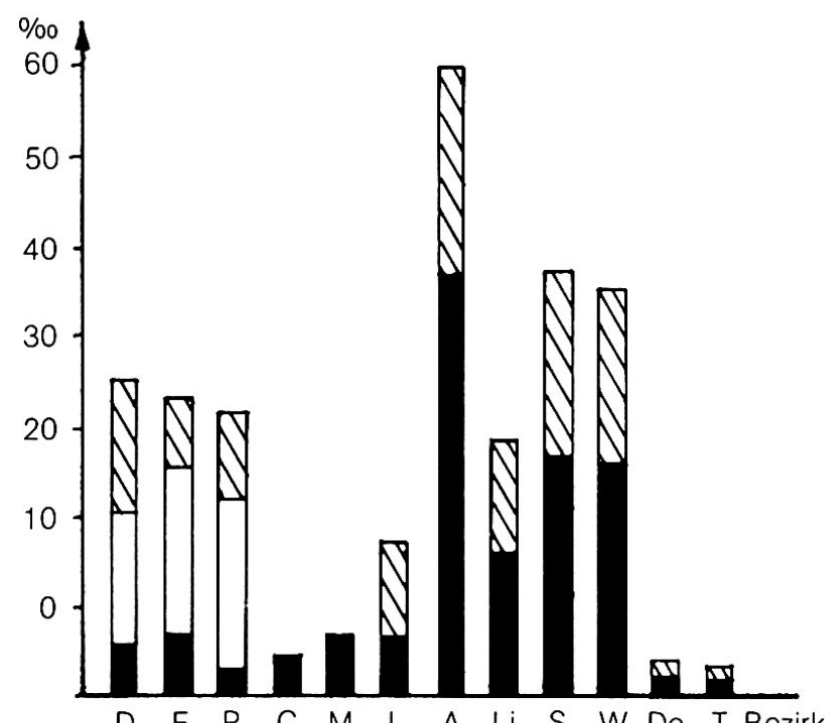

Lehrlinge

Berufsschüler

DV Fachschüler

Abkürzungen der Bezirke auf Abb. 1

Ausgebildete: männliche Schüler der Schulen Sissach-Ebenrain(1974-82), Delémont-Courtemelon (1973/74-1981/82) und Laufen (1971-1980/81), deren Eltern in obengenannten Bezirken wohnten. 
rain); Liestal weist $1,58 \%$ auf, Sissach $2,61 \%$ und Waldenburg 2,59\%. Die Fachschule besuchten 2,27\% der Landwirte aus dem Bezirk Arlesheim, 1,2\% aus Liestal, 2,05\% aus Sissach und 1,87\% aus Waldenburg. Mit Ausnahme des Bezirks Waldenburg schickten alle basellandschaftlichen Bezirke weibliche Lehrlinge in die Ausbildung und rund $1,1 \%$ der Bäuerinnen absolvierten die einjährige Fachschule (ab 18 J.)! Aus dem Laufental besuchten $0,1 \%$ die Bäuerinnenfachschule.

Folgerung: Die Nähe der Ausbildungsstätte spielt bei den (angehenden) Bauern für den Schulbesuch eine übergeordnete Rolle, was der höhere Besucheranteil aus den Schulbezirken beweist (Sissach und Delé- mont). Benachteiligt sind jene Bezirke, die durch die politische oder die Sprachgrenze von einer bedeutenden Schule (Ebenrain, Courtemelon) getrennt sind. Laufen schickte schon vor der Lostrennung des Kantons Jura kaum Schüler nach Courtemelon (École cant. d'agr. et École ménagère rurale, 1971:71) oder Ebenrain; die solothurnischen Bezirke sind auch topographisch vom Mittelland getrennt. Mit zunehmender Bildung und Verstädterung des Lebensraumes scheint zudem der Besuch landwirtschaftlicher Schulen anzusteigen, wie die Zahlen des Bezirks Arlesheim zeigen. In Courtemelon wie in Ebenrain ist zudem der Anteil der ausgebildeten jungen Leute aus finanziell gutgestelltem und nichtbäuerlichem Milieu groß.

\section{Zusammenfassung}

Es sollte ermittelt werden, ob sich der Anteil der Schulabsolventen je nach Sprachgebiet unterscheidet und ob der Schulbesuch von der Nähe einer landwirtschaftlichen Ausbildungsstätte abhängt. Erfaßt wurden fünf französisch- und sieben deutschsprachige Bezirke der Kantone Baselland, Bern, Jura und Solothurn.

Die Anzahl Absolventen wurden über einen längeren Zeitraum gemittelt und zum Durchschnitt der Landwirtschaftsbetriebe aus drei Stichjahren (1969, 1975 und 1980) gesetzt. Der Vergleich fand bezirksweise und kantonal statt und ließ weitere Schlüsse zu, z.B. auf die Nebenerwerbsbetriebe.

Die Abnahme von Landwirtschaftsbetrieben ist im französischen Sprachgebiet geringer als in den deutschsprachigen Bezirken (Baselland nimmt eine Sonderstellung ein); besonders hoch ist der Verlust im solothurnischen und bernischen Laufener Becken.
Nebenerwerbsbetriebe sind in welschen Bezirken durchwegs untervertreten und bilden offensichtlich einen gruppenspezifischen Indikator.

Die größte prozentuale Anzahl ausgebildeter Landwirte weist der Kanton Baselland auf, wobei Schüler (-innen) aus städtischem Milieu sehr zahlreich vertreten sind. Innerhalb der ländlicheren Gebiete fällt jedoch der hohe Anteil im (französischsprachigen) Kanton Jura auf. Der Schulbesuch ist zumindest auf Staatsebene auch vom Schulort abhängig, die Bezirke Sissach (Schule Ebenrain) und Delémont (Courtemelon) weisen innerhalb der Bezirke im jeweiligen Kanton die höchsten Anteile ausgebildeter Jungbauern aus. Daneben spielt aber die Sprachgrenze eine entscheidende Rolle, da schon vor der Gründung des Kantons Jura kaum Laufentaler, Thiersteiner oder Dornecker die nahegelegene, ausgezeichnete Schule im damaligen Kanton Bern besuchten als auch heute noch wenig Hindernisse für außerkantonale Schulanwärter bestehen.

\section{Literatur}

CHAPATTE G. (1972): Origine et Occupation actuelle des anciens élèves de l'Ecole d'Agriculture de Courtemelon (unveröff. Diplomarbeit), Porrentruy

Jubilé de l'Ecole cantonale d'agriculture et Ecole ménagère rurale - Courtemelon 1927-77, o. J. und o. O.
WASMER K. (1982): Die Agrarlandschaft im Raum der deutschfranzösischen Sprachgrenze im nördlichen Jura. In: Regio Basiliensis, Bd. 23, H. 1+ 2, S. 172-183.

Quellenwerke des Bundesamtes für StatistikJ Eidg. Landwirtschafts- und Gartenbauzählung, 1980; Eidg. Betriebszählung 1975 (Landwirtschaftsbetriebe); Eidg. Betriebszählung 1969 (das.) 